\title{
Design, rationale, and baseline demographics of SEARCH I: a prospective cluster-randomized study
}

This article was published in the following Dove Press journal: International Journal of COPD

10 July 2012

Number of times this article has been viewed

\section{Frank Albers' \\ Asif Shaikh² \\ Ahmar lqbal ${ }^{3}$ \\ 'Medical Affairs Respiratory, ${ }^{2}$ Clinical Development and Medical Affairs, Field Based Medicine- Respiratory, Boehringer Ingelheim Pharmaceuticals, Inc, Ridgefield, CT, USA; ${ }^{3}$ Respiratory Medical Affairs, Pfizer Inc, New York, NY, USA}

\begin{abstract}
Questionnaires are available to identify patients at risk for several chronic diseases, including COPD, but are infrequently utilized in primary care. COPD is often underdiagnosed, while at the same time the US Preventive Services Task Force recommends against spirometric screening for COPD in asymptomatic adults. Use of a symptom-based questionnaire and subsequent handheld spirometric device depending on the answers to the questionnaire is a promising approach to identify patients at risk for COPD. Screening, Evaluating and Assessing Rate CHanges of diagnosing respiratory conditions in primary care 1 (SEARCH I) was a prospective cluster-randomized study in 168 US primary care practices evaluating the effect of the COPD-Population Screener (COPD-PS ${ }^{\mathrm{TM}}$ ) questionnaire. The effect of this questionnaire alone or sequentially with the handheld copd- $6^{\mathrm{TM}}$ device was evaluated on new diagnoses of COPD and on respiratory diagnostic practice patterns (including referrals for pulmonary function testing, referrals to pulmonologists, new diagnoses of COPD, and new respiratory medication prescriptions). Participating practices entered a total of 9704 consecutive consenting subjects aged $\geq 40$ years attending primary care clinics. Study arm results were compared for new COPD diagnosis rates between usual care and (1) COPD-PS plus copd-6 and (2) COPD-PS alone. A cluster-randomization design allowed comparison of the intervention effects at the practice level instead of individuals being the subjects of the intervention. Regional principal investigators controlled the flow of study information to sub-investigators at participating practices to reduce observation bias (Hawthorne effect). The results of SEARCH I, to be published subsequently, will provide insight into the real world utility of the COPD-PS as well as two-stage COPD case finding with COPD-PS and copd-6.

Keywords: COPD Population Screener, COPD-PS ${ }^{\mathrm{TM}}$, copd-6 ${ }^{\mathrm{TM}}$, spirometry, screening, case finding
\end{abstract}

\section{Introduction}

COPD confers significant burdens of mortality, disability, health care utilization, and costs upon individuals and society. This disease now is the third leading cause of death in the $\mathrm{USA}^{1}$ and is also responsible for 670,000 hospitalizations, 16 million office visits, and US\$49.9 billion in total (direct and indirect) medical costs yearly. ${ }^{2}$ Millions of working days are lost to COPD, further worsening its financial impact., ${ }^{2,3}$

Despite the public health and economic impact of COPD, the disease remains underrecognized. Patients with early symptoms, such as exertional dyspnea, may interpret them as normal aging or minimize them by reducing activity and not seek medical attention. These factors contribute to delayed diagnoses by health care professionals despite substantial deterioration in health status and increased risk of mortality. ${ }^{4} \mathrm{Half}$ or more of US National Health and Nutrition Examination Survey III participants with
Correspondence: Frank Albers

Medical Affairs Respiratory, Boehringer Ingelheim Pharmaceuticals, Inc,

Mail Code: Corporate Center E3253, 900 Ridgebury Road, PO Box 368,

Ridgefield, CT, 06877, USA

Tel + I 9|4 $582562 \mid$

Email frank-c.albers@t-online.de 
spirometrically demonstrable airway obstruction had never previously received a diagnosis of obstructive lung disease. ${ }^{5}$ Proactive diagnosis and care of COPD is important to lessen the impact of the disease. Undiagnosed and unmanaged but progressing COPD may manifest in an acute exacerbation requiring emergency care. ${ }^{6}$ Additionally, COPD and lung cancer are closely associated beyond a simple smokingrelated link ${ }^{7}$ and the incidence of lung cancer is even higher in patients with mild-to-moderate COPD than in those with more severe COPD. ${ }^{8}$ Thus, appropriately diagnosing COPD earlier becomes even more important.

Primary care physicians (PCPs) play an important role in the initial recognition and diagnosis of COPD, as for other chronic conditions. Dyspnea, chronic cough, sputum production, and/or history of risk-factor exposure characterize patients at risk. ${ }^{9}$ Multiple professional societies' guidelines, ${ }^{9-11}$ including the Global Initiative for Chronic Obstructive Lung Disease (GOLD) ${ }^{9}$ and American College of Physicians (ACP)/American College of Chest Physicians (ACCP)/American Thoracic Society (ATS)/ European Respiratory Society (ERS) $2011^{10}$ emphasize use of spirometry to diagnose COPD. Unlike hypertension and dyslipidemia, for which PCPs and specialists alike use criterion-standard diagnostic methods (blood pressure and blood lipid measurement), a diagnostic gap exists for COPD between pulmonologists' standard (spirometry) and PCPs' frequent reliance on symptoms and clinical observations alone.

A further gap is found between respiratory societies' requirement for spirometry to diagnose COPD and the US Preventive Services Task Force's discouragement of spirometry for asymptomatic screening for COPD (despite its recognition that COPD is underdiagnosed). ${ }^{12}$ Similarly, ACP/ ACCP/ATS/ERS $2011^{10}$ states that there is "no evidence of benefit of using spirometry to screen adults who have no respiratory symptoms." However, spirometry is warranted in patients with wheezing, shortness of breath, or exertional limitations of respiratory origin and who are symptomatic. Unfortunately, patients may deny exertional limitation because they have reduced their activity to avoid dyspnea, thus limiting their reporting of symptoms to their clinician. ${ }^{10}$ Very inactive patients with unrecognized COPD may become overtly symptomatic when attempting activities normal for their age and health. ${ }^{10}$ Thus, methods are needed to assess patients' respiratory symptoms more realistically and to identify patients at risk for COPD for targeted spirometry. Studies have shown the feasibility of methods for identifying COPD patients among general or high-risk populations. ${ }^{13-15}$ The use of symptom-based questionnaires to identify patients at risk and conducting spirometry depending on patients' responses may be an appropriate and cost-effective approach.

Many questionnaires have been investigated for COPD risk identification, although their real-world PCP application has lagged behind tool development and validation. Readers are referred to a recent comparative review by Duvall and Frank ${ }^{16}$ for more details on specific questionnaires. Calverley and colleagues ${ }^{17}$ developed one of the first COPD questionnaires, which was validated retrospectively against the US National Health and Nutrition Examination Survey III database. Price and colleagues ${ }^{18}$ prospectively developed a questionnaire to identify COPD risk in smokers not previously diagnosed with respiratory diseases; a subsequent Dutch study ${ }^{19}$ used the Price questionnaire by telephone to stratify smokers by COPD risk and invite those at medium or high risk for case-finding spirometry. Hanania et $\mathrm{a}^{20}$ developed and validated the Lung Function Questionnaire. When tested in primary care practices, this questionnaire had a sensitivity of $82.6 \%$ and specificity of $47.8 \%$, with $54.3 \%$ correctly classified as with or without COPD based on pre- and post-bronchodilator spirometry and the then-current GOLD criteria. ${ }^{20}$ The Clinical COPD Questionnaire ${ }^{21}$ is designed primarily to measure symptoms, function, and clinical control in patients already diagnosed with COPD; however, it was used as an initial questionnaire together with the Medical Research Council dyspnea scale in a Swedish primary care COPD screening study. ${ }^{22}$ Other COPD patient identification tools include the COPD Assessment Questionnaire ${ }^{23}$ and the COPD Population Screener (COPD-PSTM, a trademark of Quality Metric Incorporated). ${ }^{24}$

The COPD-PS assesses dyspnea, phlegm, reduced activity because of breathing problems, smoking ( $\geq 100$ cigarettes lifelong), and age group. Scores $\geq 5$ identify patients at risk of COPD with a sensitivity of $84.4 \%$, specificity of $60.7 \%$, and positive predictive value of $56.8 \% .{ }^{24}$ The COPD-PS has been validated in English ${ }^{24}$ and Spanish, ${ }^{25}$ in paper $^{24}$ and Webbased versions, ${ }^{26}$ and with unselected patients presenting to PCPs and respiratory clinics ${ }^{24}$ and media-recruited members of the general public. ${ }^{26}$ This tool is familiar to the authors and was used in SEARCH I to gain insight into its applicability in real-world practice.

Two-stage screening (first step, questionnaire; second step for those at risk, handheld spirometric device, followed by full diagnostic spirometry for those with abnormal handheld device measurements) has been advocated ${ }^{27}$ to identify patients with or at risk for COPD. Possible advantages of 
this approach include reduced testing costs,${ }^{22}$ fewer false positives than with unselected screening spirometry, ${ }^{27}$ and compatibility with US Preventive Services Task Force recommendations. ${ }^{12}$ The second step for patients with at-risk questionnaire scores may measure peak expiratory flow (PEF) or forced expiratory volume in 1 second/6 seconds $\left(\mathrm{FEV}_{1} / \mathrm{FEV}_{6}\right)$. A study questioning patients on six risk factors, then measuring PEF for those at risk, referred 5\% of 5323 presenting volunteers for full spirometry (those with $\geq 2$ risk factors and a PEF $<70 \%){ }^{20} \mathrm{PEF}$ is more variable than $\mathrm{FEV}_{1}{ }^{28}$ thus may not be an optimal second stage. ${ }^{29}$ Alternatively, a handheld spirometric device can be used to measure $\mathrm{FEV}_{1} / \mathrm{FEV}_{6}$. $\mathrm{FEV}_{6}$ has been shown to be a satisfactory surrogate for forced vital capacity (FVC) that is easier to measure than FVC under field conditions or in impaired/elderly patients. ${ }^{30-35}$ Handheld devices for $\mathrm{FEV}_{1} /$ $\mathrm{FEV}_{6}$ measurements include the nSpire PiKo- ${ }^{\mathrm{TM}}$ (nSpire Health Inc, Longmont, CO) and Vitalograph ${ }^{\circledR}$ copd- $6^{\mathrm{TM}}$ (Lenexa, KS), which have both been validated against regular pulmonary function testing. ${ }^{36-39}$ PiKo-6 stores multiple sessions' measurements and is designed for home use by one patient, whereas the copd- 6 device is designed for ease of use in a practice working with multiple patients daily (eg, it has single-use disposable mouthpieces with a one-way valve to prevent cross contamination). Thus, the copd-6 was considered more suitable for the primary care setting and was selected for the current study. This device has been evaluated in primary care studies in Greece, ${ }^{37}$ Spain, ${ }^{38}$ and Sweden. ${ }^{22}$

The Screening, Evaluating, and Assessing Rate CHanges of diagnosing respiratory conditions in primary care (SEARCH I) study is designed and initiated to provide insights into whether screening tools (COPD-PS with or without subsequent use of copd-6 to identify patients at risk for COPD) can improve COPD diagnosis rates in routine primary care clinical practice. The aims of this paper are to describe the background and rationale behind the SEARCH I study design, the study design and methods, baseline data (patients' demographics and reasons for index visits), and the questions SEARCH I is designed to answer.

\section{SEARCH I study objectives}

The objectives of SEARCH I were to:

- determine the impact of a screening and case-finding tool set consisting of the COPD-PS followed by use of the copd-6 in those patients with a COPD-PS score of $\geq 5$, in comparison with usual care alone on the diagnostic yield of COPD in the primary care setting
- determine the impact of the COPD-PS (questionnaire alone without copd-6 use) in comparison with usual care alone on the diagnostic yield of COPD in the primary care setting

- explore physicians' actions after implementation of COPD-PS with or without copd-6 in a primary care setting.

\section{Methods Study design and ethics}

SEARCH I was a multicenter prospective cluster-randomized study consisting of three study arms: (1) use of COPD-PS plus copd-6, (2) use of COPD-PS alone, and (3) usual care. Because the direct objects of intervention were not patients but individual participating primary care practices (sites), these were the units of randomization, irrespective of the number of PCPs at a site. However, the number of enrolled patients per site was limited to avoid bias from any one particular site contributing a large share of the study patients. Since the practice sites were the object of the intervention, the study process utilized regional investigators who implemented the study at the primary care sites to reduce observational bias. The practice sites thus were not specifically informed about the study interventions (particularly about those of the other two arms) or the effects being studied. Also, no additional education on COPD management was provided to participating clinics. The protocol and informed consent materials received approval from central and local institutional review boards. The visit flow chart is shown in Table 1.

Table I Data collection schedule

\begin{tabular}{|c|c|c|}
\hline \multicolumn{3}{|l|}{ Study period } \\
\hline Visit & 1 & Follow-up \\
\hline Day & 1 & $\geq 56$ \\
\hline Informed consent & $x$ & \\
\hline Inclusion criteria & $x$ & \\
\hline Demographics & $x$ & \\
\hline Smoking history & $x$ & \\
\hline Concomitant diagnoses ${ }^{\mathrm{a}}$ & $x$ & \\
\hline Respiratory medications & $x$ & $x$ \\
\hline COPD-PS TM questionnaire (Arm I and Arm 2) & $x$ & \\
\hline copd-6 ${ }^{\mathrm{TM}}$ measurements (Arm I) & $x$ & \\
\hline Serious adverse events & $x$ & $x$ \\
\hline Medical chart review ${ }^{b}$ & & $x$ \\
\hline Termination of study & & $x$ \\
\hline
\end{tabular}

Notes: aRecorded from the medical chart problem list at Visit I. The problem list had to be copied at Visit I prior to the subject office visit with the sub-investigator; ${ }^{b}$ For collecting data on the endpoints, beginning 8 weeks after the last entered subject completed Visit I at each site; the information via medical chart review was recorded for the 8-week period following Visit I (includes Visit I). Abbreviation: COPD-PS, COPD Population Screener. 


\section{Subjects}

Each site screened and entered consecutive eligible patients who presented themselves for scheduled primary care visits. Eligible patients were consenting men and women $\geq 40$ years of age, literate in English or Spanish. Patients who, in the judgment of site investigators, could not perform arm-specific study procedures were excluded. Enrollment was planned to enter 55 patients per site (across 171 sites) for a total of 9405 patients. Site limitations on enrollment were intended to minimize recruitment bias from disproportionate contributions of any one specific site to the sample. Ultimately, enrollment was expanded to enter 55 patients per site who were not previously diagnosed with COPD according to the site investigator.

\section{Interventions}

The study interventions consisted only of practice use of the COPD-PS with or without the copd-6. The participating sites were aware that the study was investigating the diagnosis of respiratory conditions in primary care and that there were three arms in the study but were not informed of details of the other two arms in which they were not participating. Restricting the practices' awareness of other study arms' interventions was important to reduce a nonspecific influence of study participation on their usual care patterns (Hawthorne effect). Also, sites did not receive any specific additional education about COPD or training on the diagnosis of COPD, as this would have introduced an additional variable and would have interfered with the real-life approach of the study. Post-randomization, sub-investigators at the sites received an arm-specific study description following the concepts of the protocol that provided the background/objective for the study. Regionally located principal investigators had knowledge of all arms of the study protocol and interfaced in a consistent manner with the sub-investigators and their staffs. Randomization of sites to arms was regionally stratified.

Enrolling primary care practices were randomized to one of the three arms:

- Arm 1: COPD-PS plus copd-6.

- Practices randomized to Arm 1 implemented use of both study screening tools and were advised to follow their usual care processes subsequent to use of the tools. Patients answered paper COPD-PS questionnaires and those who scored $\geq 5$ were also evaluated with copd- 6 .

- Arm 2: COPD-PS alone.

- Practices randomized to Arm 2 implemented use of COPD-PS but not copd-6 and were advised to follow their usual care processes subsequent to use of COPD-PS.
- Arm 3: Usual care.

- Practices randomized to Arm 3 did not receive information about the screening tool(s) and were advised to follow their usual care practices.

\section{Data collection and outcome measurements}

Outcomes were evaluated beginning 8 weeks after the last subject's entry at each site by reviewing medical charts during the 8-week period including Visit 1 to collect data on the endpoints. Clinical diagnoses of COPD were determined from physicians' chart notes and new diagnoses of COPD were determined by comparison of physicians' chart notes with the problem list in the subject's medical chart.

The nested co-primary endpoints (evaluated by hierarchical closed-testing procedure) were: (1) the diagnostic yield of COPD in Arm 1 (COPD-PS + copd-6) versus Arm 3 (usual care) and (2) the diagnostic yield of COPD in Arm 2 (COPD-PS alone) versus Arm 3 (usual care). Diagnostic yield was determined for each site as the proportion of patients with a new clinical diagnosis of COPD out of those patients without a prior diagnosis of COPD.

Documentation of the clinical diagnosis of COPD in study subjects within 8 weeks of Visit 1 , ascertained by review of the medical chart of the subject at the primary care site, was the measure to determine the primary endpoint of diagnostic yield. This is based on the methodology adopted by the Research and Development (RAND) Corporation in its appropriateness of care studies to document diagnoses of conditions in a subject's medical chart at the physician's office. ${ }^{41}$ The physician documentation of the diagnosis of a condition is considered as a reliable and credible source of information for this type of data, with the understanding that information used by the physician in arriving at the diagnosis may not always be readily available in the medical chart. ${ }^{40-42}$ This is especially so for COPD, where spirometry results may not be documented, or spirometry may not even be performed, in some subjects with a clinical diagnosis of COPD. It was expected that the 8-week follow-up period including Visit 1 would be sufficient to allow for the scheduling of referrals for pulmonary function test (PFT) or pulmonologist consultation (if ordered at Visit 1) and, in most cases, to receive, review, and record the relevant information from those referrals in the medical chart.

Secondary endpoints compared the diagnostic practice pattern of COPD assessed from physicians' notes in medical charts within the 8-week period including Visit 1 (proportion of consenting and previously undiagnosed subjects with either a new clinical diagnosis of COPD assigned at Visit 1, 
a referral to pulmonary function testing or to a pulmonologist, or a prescription of new respiratory medication) between Arms 1 and 3 and between Arms 2 and 3.

The diagnostic practice pattern endpoint assessed the impact of the screening tool(s) (COPD-PS alone or COPD-PS and copd-6) on physician actions related to a possible clinical diagnosis of COPD.

Safety was assessed by any serious adverse events reported with study-related procedures and any serious adverse events reported by subjects taking Boehringer Ingelheim (Ridgefield, $\mathrm{CT}$ ) products.

\section{Statistical methods}

Primary and secondary endpoints per site were derived from the data of all consenting entered subjects without a prior diagnosis of COPD. Analysis of the primary and secondary endpoints per site accounted for the effects of site-based clustering of subjects, which are inherent in a cluster-randomized study design.

The primary endpoint is to be evaluated using an analysis of variance including the fixed effects of intervention arm and region. To account for differing numbers of patients at each site with no prior diagnosis of COPD, the analysis will be appropriately weighted and adjusted for the number of patients per site. The following hierarchical closed testing procedure will control for Type I experiment-wise error and thus utilize a one-sided alpha equal to $0.025 .^{43}$ An initial test will determine superiority of Arm 1 versus Arm 3 in the diagnostic yield of newly diagnosed COPD. If this initial test is significant, then the superiority of Arm 2 versus Arm 3 in the diagnostic yield of newly diagnosed COPD is to be determined in the same way; if the initial test is not significant, the Arm 2 versus Arm 3 comparison will be descriptive only. Comparison results will be expressed as adjusted least-squares mean differences between arms with standard errors, $95 \%$ confidence intervals (CIs), and $P$ values; significant differences will be recognized as lower CI $>0$ with level of significance given by the respective $P$ values.

To assess whether intervention arm effects are homogenous across regions, a sensitivity analysis is planned using the statistical model used in the primary analysis but also including a term for any arm-by-region interaction. To account for differences in the number of primary care sites per intervention arm and region, the Type II sum of squares (using PROC GLM in SAS ${ }^{\circledR}$ software, v 9.2; SAS Institute, Cary, $\mathrm{NC}$ ) is to be used in this analysis for comparing effects of intervention arms.
An additional sensitivity/confirmatory analysis is planned, fitting a negative binomial distribution to the site counts of newly diagnosed COPD patients, accounting for differing numbers of patients at each site with no prior diagnosis. As well, an analysis at the patient level is planned to analyze the logits of the binary variable of new diagnosis of COPD (or not), including in the statistical model a random effect to account for the clustering effect inherent in the study design.

The secondary endpoint of diagnostic practice pattern will be analyzed using the same analysis of variance model described for the primary endpoint and utilize a one-sided 0.025 level of significance.

Summary statistics will be computed for continuous variables, with frequencies ( $\mathrm{N} \%)$ given for categorical variables.

\section{Rationale for sample size}

Enrollment was planned to include 171 primary care sites (clusters) to be randomized 1:1:1 to the study arms (57 sites per arm; 55 subjects with no prior diagnosis of COPD per site, for a total of 9405 subjects [3135 subjects per arm]). Planning of sample size and analysis procedures took into account the correlations among observations within sites expected with cluster randomization. The intracluster correlation coefficient (ICC; $\rho$ ) captured the proportion of the total (between + within) variability attributable to primary care sites (clusters). This coefficient, along with the cluster size, determined the design effect. The number of subjects evaluated at each PCP site (cluster) represented the cluster size $(\mathrm{m})$ : design effect $=[1+(m-1) \times \rho]$. The ICC value for this study was estimated at 0.0185 , which was the median ICC value of all variables studied in a similarly designed cluster-randomized diabetes study of Vermont primary care practices ${ }^{44}$ yielding an assumed cluster design effect of 1.999.

Diagnostic yield of COPD in primary care practices is not known and may show geographic or other variability. In a National Committee for Quality Assurance survey of new COPD diagnoses in adults over 40 across five diverse health insurers, ${ }^{45}$ diagnostic yield found an average of $0.3 \%$ with a range from $0.25 \%$ to $1.57 \%$. Based on this data, a diagnostic yield of $1.5 \%$ was assumed for the usual care intervention arm (Arm 3). Assuming that intervention with the COPD-PS and COPD 6 (Arm 1) will result in an increased diagnostic yield of COPD compared with intervention with COPD-PS alone (Arm 2), sample size was calculated for adequate power to detect a difference when comparing Arm 2 and Arm 3, which also assures adequate 
power for comparing Arm 1 and Arm 3. Since the COPD-PS has a positive-predictive value of about $56 \%$, and assuming COPD prevalence is $5.5 \%$, the use of the COPD-PS alone could result in a diagnostic yield of $3 \%$, translating into a $1.5 \%$ difference in diagnostic yield compared with the assumption of $1.5 \%$ diagnostic yield in the usual care arm (Arm 3). Table 2 shows the sample size calculations resulting from the assumptions discussed.

\section{Study demographics and reasons for index visits}

\section{Demographics and prior diagnoses of COPD}

Table 3 shows the entered practices and subjects by region. Actual enrollment from 168 primary care practices was 9754 subjects with 9704 entered into the study; 8770 with no prior diagnosis of COPD. For the 8770 subjects with no prior diagnosis of COPD, the mean age was $60.2 \pm 12.6$ years; there were 5211 women (59.4\%) and 3559 men (40.6\%). Ethnic proportions were $82.9 \%$ Caucasian, $10.2 \%$ AfricanAmerican, 5.4\% Asian, 0.9\% Native American/Inuit, 0.4\% Hawaiian/Pacific Islander, with $0.1 \%$ not responding; $19.6 \%$ of participants were Hispanic/Latino, 79.5\% non-Hispanic, with $0.9 \%$ not responding. Smoking history showed $17.2 \%$ current smokers, $31.8 \%$ ex-smokers, and $50.1 \%$ nonsmokers, with $0.9 \%$ not responding.

\section{Index visits}

Subjects' reasons for index visits were: annual physical, $24.4 \%$; respiratory issues, $3.7 \%$; other, $70.4 \%$; with $1.5 \%$ not responding.

Table 2 Total sample size for various scenarios, assuming a diagnostic yield rate for control group to be $1.5 \%$, the rate for treatment group to be $3 \%$ and intracluster correlation coefficient $=0.0185^{\mathrm{a}}$

\begin{tabular}{|c|c|c|c|c|c|c|}
\hline \multirow{2}{*}{$\begin{array}{l}\text { Patients } \\
\text { per site }\end{array}$} & \multicolumn{2}{|c|}{$90 \%$ power } & \multicolumn{2}{|c|}{$85 \%$ power } & \multicolumn{2}{|c|}{$80 \%$ power } \\
\hline & $\begin{array}{l}\text { Total } \\
\text { patients }\end{array}$ & $\begin{array}{l}\text { Total } \\
\text { sites }\end{array}$ & $\begin{array}{l}\text { Total } \\
\text { patients }\end{array}$ & $\begin{array}{l}\text { Total } \\
\text { sites }\end{array}$ & $\begin{array}{l}\text { Total } \\
\text { patients }\end{array}$ & $\begin{array}{l}\text { Total } \\
\text { sites }\end{array}$ \\
\hline 50 & 11,850 & 237 & 10,200 & 204 & 9000 & 180 \\
\hline 55 & 12,540 & 228 & 10,725 & 195 & 9405 & 171 \\
\hline 60 & 13,140 & 219 & 11,160 & 186 & 9720 & 162 \\
\hline 65 & 13,650 & 210 & 11,700 & 180 & 10,335 & 159 \\
\hline 70 & 14,280 & 204 & 12,180 & 174 & 10,710 & 153 \\
\hline 75 & 14,850 & 198 & 12,600 & 168 & 11,025 & 147 \\
\hline 80 & 15,360 & 192 & 13,200 & 165 & 11,520 & 144 \\
\hline 85 & 16,065 & 189 & 13,770 & 162 & 11,985 & $14 \mid$ \\
\hline 90 & 16,470 & 183 & 14,310 & 159 & 12,420 & 138 \\
\hline 95 & 17,100 & 180 & 14,820 & 156 & 12,825 & 135 \\
\hline 100 & 17,700 & 177 & 15,300 & 153 & 13,200 & 132 \\
\hline
\end{tabular}

Note: abased on sample size calculations using PROC POWER in SAS ${ }^{\circledR}$ (v 9.2; SAS Institute, Cary, NC).
Table 3 Subjects and primary care practices entered into the study by region as of January 31,2012

\begin{tabular}{lll}
\hline Region & $\begin{array}{l}\text { Number of } \\
\text { centers }\end{array}$ & $\begin{array}{l}\text { Number of } \\
\text { patients entered }\end{array}$ \\
\hline St Petersburg, FL & 27 & 1648 \\
Kentucky & 14 & 884 \\
San Francisco, CA & 36 & 2027 \\
Virginia & 9 & 451 \\
Minnesota & 15 & 875 \\
Oregon & 21 & 1287 \\
Michigan & 11 & 555 \\
Miami, FL & 17 & 979 \\
Los Angeles, CA & 18 & 998 \\
Total & 168 & 9704 \\
\hline
\end{tabular}

\section{Discussion}

SEARCH I was a real-world study in primary care clinics, evaluating the effect of the COPD-PS questionnaire used both with and without the copd- 6 handheld spirometer compared with usual care on COPD diagnosis rates and practice patterns. Because the evaluations of interest were changes in diagnostic rates and practice patterns, SEARCH I used a cluster design in which the PCP practice, not the subject, was the unit of randomization. To represent general practice patient populations, SEARCH I consecutively enrolled consenting consecutive patients aged $\geq 40$ years presenting for primary care appointments. A total of 9704 subjects from 168 practices participated, with considerable geographic diversity across the USA. The last subject's entry to SEARCH I occurred on August 5, 2011, chart abstracting was completed on October 28, 2011, and database lock occurred on December 2, 2011. Results will be presented at congresses in 2012/13 and published in a primary paper.

Spirometric screening of all asymptomatic adults for COPD is expensive and is discouraged by the US Preventive Services Task Force ${ }^{12}$ and the 2011 ACP/ACCP/ATS/ ERS guidelines..$^{10}$ Conversely, focusing on specific subsets of patients (eg, current smokers or elderly smokers) may miss other patients with unrecognized COPD in the general population. ${ }^{46}$ Two-stage screening using a symptomatic questionnaire and an inexpensive handheld device is expected to reduce costs of case finding (the cost of the copd- 6 device is about one-tenth that of an office spirometer ${ }^{47}$ ). Two-stage screening identifies candidates for full spirometry who have a high pre-test probability of having $\mathrm{COPD}^{48,49}$ and can be implemented with a follow-up visit for diagnosis and management plan for COPD. ${ }^{9}$ Thus, SEARCH I employed the COPD-PS symptom-based questionnaire for initial identification of subjects at risk for COPD, either on its 
own or followed by copd-6 handheld spirometry in a twostage screening protocol to identify candidates for a clinical diagnosis of COPD that should be followed by a spirometric diagnosis. SEARCH I assessed effects of these COPD screening tools in day-to-day practice within a real-world primary care patient population.

SEARCH I was designed to answer two questions: (1) How does use of COPD-PS followed by copd-6 or use of COPD-PS alone, as compared with usual care, affect diagnostic yield of COPD in consecutive unselected primary care subjects with no prior diagnosis of COPD and $\geq 40$ years of age? (2) What actions (assigning a clinical COPD diagnosis at the same visit, referring subjects for pulmonary function testing and/or to pulmonologists, or prescribing respiratory medications) do clinicians engage in after using COPD-PS with or without subsequent copd-6 as compared with usual care?

Each aspect of the study design was intended to preempt a potential difficulty. The study structure built around regional principal investigators was intended to reduce the Hawthorne effect and guard against observation bias. Regional investigators controlled the protocol information available to participating practices; each practice knew generally that this was a study of respiratory diagnosis practice patterns but was not aware of the practice interventions applied in study arms other than its own. To minimize interference with the real-life approach of the study, participating primary care sites did not receive any specific additional education about COPD and training on the diagnosis of COPD. New clinical diagnoses of COPD were ascertained by medical record abstraction (the RAND Corporation criterion standard method). The 8-week time frame, including the day of the patient visit for chart review, was based on a consensus of the time required for practice-based interventions to show an effect. Participants determined to have a prior diagnosis of COPD according to the site investigator were excluded from the denominator when proportions of newly SEARCH I-diagnosed subjects were computed. The secondary endpoint of diagnostic practice pattern comprised multiple surrogates for a clinical diagnosis of COPD - not only diagnosis itself at the initial visit but also referrals to pulmonary function testing or pulmonologist visits as well as new prescriptions of respiratory medications within 8 weeks following Visit 1 .

Potential limitations of SEARCH I include the lack of protocol-mandated follow-up spirometry for subjects receiving new clinical diagnoses of COPD. It can be expected that from one-third to possibly one-half of the subjects with a clinical diagnosis of COPD will receive spirometry. ${ }^{50}$ However, mandatory follow-up spirometry would have multiplied logistical challenges - for example, maintaining subjects' participation beyond the 8 weeks until medical records had been abstracted - as opposed to conducting subjects' study procedures only at Visit 1 . The choice of an 8-week window from Visit 1 to the follow-up medical record abstraction to identify a new clinical diagnosis assumed that 8 weeks would suffice for subjects to attend an appointment for spirometry and the primary care provider to receive and chart the results. A longer time window would have allowed for longer delays in referral appointments. Finally, no prior-year historic data have been collected from participating sites, so any potential changes of practice in the usual care comparison arm could not have been detected.

\section{Conclusion}

COPD is frequently underdiagnosed or misdiagnosed. ${ }^{14,51-53}$ A simple, self-administered, self-scored, validated tool in the form of a questionnaire to identify individuals at risk for COPD can increase awareness and earlier symptom recognition by patients and physicians. The subsequent use of a handheld spirometer in those patients identified as at risk for COPD could be a feasible and effective way to detect patients with airway obstruction, facilitating definitive spirometric diagnosis and addressing the gap in primary care in the diagnostic approach to undetected COPD. Implementation of such a two-stage screening process in the primary care community would assist physicians in identifying individuals at risk for COPD who should be considered for a clinical diagnosis of COPD and would need a diagnostic spirometry follow-up assessment. PCPs have an opportunity to initiate recognition and proactive management of COPD, striving for earlier smoking cessation, reduced exacerbations, and improved outcomes. Results from SEARCH I will provide data on the realworld impact of the COPD-PS and copd-6 screening tools on COPD diagnosis and management in 168 primary care practices in multiple US regions.

\section{Acknowledgments}

This study is supported by Boehringer Ingelheim and Pfizer. The Principal Investigator is Dr William C Wadland; regional investigators are Drs Anthony Baldizzi, Michael Campos, David M Mannino, John Peabody, Edmundo Rubio, Barbara Yawn, and Karen Duvall. Drs Thomas Leonard, Christine Baker, Jun Su, and Santosh Sutradhar have contributed to study design and/or analysis. Stephen Koval provided statistical analysis. Under the authors' conceptual direction, 
medical writing assistance for this paper was provided by Kim Coleman Healy, PhD, CMPP, of UBC-Envision Group; medical writing assistance was supported by Boehringer Ingelheim and Pfizer Inc.

\section{Disclosure}

Frank Albers was an employee of Boehringer Ingelheim during the study and at manuscript acceptance. Asif Shaikh is an employee of Boehringer Ingelheim and Ahmar Iqbal is an employee of Pfizer Inc.

\section{References}

1. Kleinschmidt P. Chronic Obstructive Pulmonary Disease and Emphysema in Emergency Medicine. Medscape Reference. October 10, 2011. Available from: http://emedicine.medscape.com/article/807143overview\#showall. Accessed November 1, 2011.

2. US National Heart Lung and Blood Institute. Morbidity and Mortality: the 2009 Chart Book on Cardiovascular, Lung, and Blood Diseases. Bethesda, MD: National Institutes of Health; 2009.

3. Parkes G, Greenhalgh T, Griffin M, Dent R. Effect on smoking quit rate of telling patients their lung age: the Step2quit randomised controlled trial. BMJ. 2008;336(7644):598-600.

4. van Schayck CP, Chavannes CN. Detection of asthma and chronic obstructive pulmonary disease in primary care. Eur Respir J. 2003; 21(Suppl 39):16S-22S.

5. Mannino DM, Gagnon RC, Petty TL, Lydick E. Obstructive lung disease and low lung function in adults in the United States: data from the National Health and Nutrition Examination Survey, 1988-1994. Arch Intern Med. 2000;160(11):1683-1689.

6. Calverley PM. COPD: early detection and intervention. Chest. 2000; 117(5 Suppl 2):365S-371S.

7. Barnes PJ, Adcock IM. Chronic obstructive pulmonary disease and lung cancer: a lethal association. Am J Respir Crit Care Med. 2011;184(8): $866-867$.

8. de Torres JP, Marin JM, Casanova C, et al. Lung cancer in patients with COPD: Incidence and predicting factors. Am J Respir Crit Care Med. Epub July 28, 2011.

9. Global Initiative for Chronic Obstructive Lung Disease. Global Strategy for the Diagnosis, Management and Prevention of COPD. Global Initiative for Chronic Obstructive Lung Disease; 2010. Available from: http://www.goldcopd.org/guidelines-global-strategy-for-diagnosismanagement.html. Accessed July 25, 2011.

10. Qaseem A, Wilt TJ, Weinberger SE, et al. Diagnosis and management of stable chronic obstructive pulmonary disease: a clinical practice guideline update from the American College of Physicians, American College of Chest Physicians, American Thoracic Society, and European Respiratory Society. Ann Intern Med. 2011;155(3):179-191.

11. American Thoracic Society, European Respiratory Society. Standards for the Diagnosis and Management of Patients with COPD. New York, NY: American Thoracic Society; 2004. Available from: http://www. thoracic.org/clinical/copd-guidelines/index.php. Accessed May 17, 2012.

12. Screening for chronic obstructive pulmonary disease using spirometry: US Preventive Services Task Force recommendation statement. Ann Intern Med. 2008;148(7):529-534.

13. van den Boom G, van Schayck CP, van Möllen MP, et al. Active detection of chronic obstructive pulmonary disease and asthma in the general population. Results and economic consequences of the DIMCA program. Am J Respir Crit Care Med. 1998;158(6):1730-1738.

14. Zielinski J, Bednarek M. Early detection of COPD in a high-risk population using spirometric screening. Chest. 2001;119(3): $731-736$.
15. Tirimanna PR, van Schayck CP, den Otter JJ, et al. Prevalence of asthma and COPD in general practice in 1992: has it changed since 1977? $\mathrm{Br}$ J Gen Pract. 1996;46(406):277-281.

16. Duvall K, Frank GW. Identifying chronic obstructive pulmonary disease in primary care of urban underserved patients: tools, applications, and challenges. J Natl Med Assoc. 2010;102(7):570-578.

17. Calverley PM, Nordyke RJ, Halbert RJ, Isonaka S, Nonikov D. Development of a population-based screening questionnaire for COPD. COPD. 2005;2(2):225-232.

18. Price DB, Tinkelman DG, Halbert RJ, et al. Symptom-based questionnaire for identifying COPD in smokers. Respiration. 2006; 73(3):285-295.

19. Dirven JA, Muris JW, van Schayck CP. COPD screening in general practice using a telephone questionnaire. COPD. 2010;7(5):352-359.

20. Hanania NA, Mannino DM, Yawn BP, et al. Predicting risk of airflow obstruction in primary care: Validation of the lung function questionnaire (LFQ). Respir Med. 2010;104(8):1160-1170.

21. van der Molen T, Willemse BW, Schokker S, ten Hacken NH, Postma DS, Juniper EF. Development, validity and responsiveness of the Clinical COPD Questionnaire. Health Qual Life Outcomes. 2003; $1: 13$.

22. Thorn J, Tilling B, Lisspers K, Jorgensen L, Stenling A, Stratelis G. Improved prediction of COPD in at-risk patients using lung function pre-screening in primary care: a real-life study and cost-effectiveness analysis. Prim Care Respir J. Epub Jan 23, 2012.

23. Bailey WC, Sciurba FC, Hanania NA, et al. Development and validation of the Chronic Obstructive Pulmonary Disease Assessment Questionnaire (COPD-AQ). Prim Care Respir J. 2009;18(3):198-207.

24. Martinez FJ, Raczek AE, Seifer FD, et al. Development and initial validation of a self-scored COPD Population Screener Questionnaire (COPD-PS). COPD. 2008;5(2):85-95.

25. Miravitlles M, Llor C, Calvo E, Díaz S, Díaz-Cuervo H, GonzalezRojas N. Validation of the Spanish version of the Chronic Obstructive Pulmonary Disease-Population Screener (COPD-PS). Its usefulness and that of FEV(1)/FEV(6) for the diagnosis of COPD. Med Clin (Barc). Epub October 18, 2011. Spanish.

26. Beaumont JL, Victorson D, Su J, et al. Examining web equivalence and risk factor sensitivity of the COPD population screener. Value Health. 2011;14(4):506-512.

27. Enright P. Provide GPs with spirometry, not spirometers. Thorax. 2008;63(5):387-388.

28. Chan-Yeung M, Carlsten C. Reasonable alternatives to spirometry for diagnosing chronic obstructive pulmonary disease: is the peak flow meter the answer? Int J Tuberc Lung Dis. 2009;13(3):279-280.

29. Perez-Padilla R, Vollmer WM, Vázquez-García JC, Enright PL, Menezes AM, Buist AS; BOLD and PLATINO Study Groups. Can a normal peak expiratory flow exclude severe chronic obstructive pulmonary disease? Int J Tuberc Lung Dis. 2009;13(3):387-393.

30. Gleeson S, Mitchell B, Pasquarella C, Reardon E, Falsone J, Berman L. Comparison of $\mathrm{FEV}_{6}$ and $\mathrm{FVC}$ for detection of airway obstruction in a community hospital pulmonary function laboratory. Respir Med. 2006;100(8):1397-1401.

31. Melbye $\mathrm{H}$, Medbø A, Crockett $\mathrm{A}$. The $\mathrm{FEV}_{1} / \mathrm{FEV}_{6}$ ratio is a good substitute for the $\mathrm{FEV}_{1} / \mathrm{FVC}$ ratio in the elderly. Prim Care Respir J. 2006;15(5):294-298.

32. Bellia V, Sorino C, Catalano F, et al. Validation of $\mathrm{FEV}_{6}$ in the elderly: correlates of performance and repeatability. Thorax. 2008;63(1): 60-66.

33. Sorino C, Sherrill D, Guerra S, et al. Prognostic value of $\mathrm{FEV}_{1} / \mathrm{FEV}_{6}$ in elderly people. Clin Physiol Funct Imaging. 2011;31(2):101-107.

34. Rosa FW, Perez-Padilla R, Camelier A, Nascimento OA, Menezes AM, Jardim JR; Latin American Project for Investigation of Obstructive Lung Disease (PLATINO) Group. Efficacy of the $\mathrm{FEV}_{1} / \mathrm{FEV}_{6}$ ratio compared to the $\mathrm{FEV}_{1} / \mathrm{FVC}$ ratio for the diagnosis of airway obstruction in subjects aged 40 years or over. Braz J Med Biol Res. 2007;40(12):1615-1621. 
35. Jing JY, Huang TC, Cui W, Xu F, Shen $\mathrm{HH}$. Should FEV $/ \mathrm{FEV}_{6}$ replace $\mathrm{FEV}_{1} / \mathrm{FVC}$ ratio to detect airway obstruction? A metaanalysis. Chest. 2009;135(4):991-998.

36. Frith P, Crockett A, Beilby J, et al. Simplified COPD screening: validation of the PiKo- $6^{\circledR}$ in primary care. Prim Care Respir J. 2011; 20(2):190-198, 2 p following 198.

37. Sichletidis L, Spyratos D, Papaioannou M, et al. A combination of the IPAG questionnaire and PiKo- $6{ }^{\circledR}$ flow meter is a valuable screening tool for COPD in the primary care setting. Prim Care Respir J. 2011;20(2): 184-189, 1 p following 189.

38. Represas Represas C, Botana Rial M, Leiro Fernández V, González Silva AI, del Campo Pérez V, Fernández-Villar A. Assessment of the portable copd-6 device for detecting obstructive airway diseases. Arch Bronconeumol. 2010;46(8):426-432. Spanish.

39. Gil-Guillén V, Orozco-Beltrán D, Carratala Munuera CV, et al; FUMEPOC Study Research Team. FUMEPOC: early detection of chronic obstructive pulmonary disease in smokers. BMC Public Health. 2011;11:413.

40. Mularski RA, Asch SM, Shrank WH, et al. The quality of obstructive lung disease care for adults in the United States as measured by adherence to recommended processes. Chest. 2006;130(6):1844-1850.

41. Roth CP, Kahn KL, Sherwood MJ, Rubenstein LV, Chew CF, Brook RH Medical Record Abstraction Form and Guidelines for Assessing Quality of Care for Hospitalized Patients with Pneumonia. Santa Monica, CA: RAND Corporation; 1988. Publication N-2801-HCFA.

42. Murata PJ, McGlynn EA, Siu AL, et al. Quality measures for prenatal care. A comparison of care in six health care plans. Arch Fam Med. 1994;3(1):41-49.

43. Bauer P, Röhmel J, Maurer W, Hothorn L. Testing strategies in multidose experiments including active control. Stat Med. 1998;17(18): 2133-2146.
44. Littenberg B, MacLean CD. Intra-cluster correlation coefficients in adults with diabetes in primary care practices: the Vermont Diabetes Information System field survey. BMC Med Res Methodol. 2006; $6: 20$.

45. Han MK, Kim MG, Mardon R, et al. Spirometry utilization for COPD: how do we measure up? Chest. 2007;132(2):403-409.

46. de Marco R, Accordini S, Cerveri I, et al. An international survey of chronic obstructive pulmonary disease in young adults according to GOLD stages. Thorax. 2004;59(2):120-125.

47. National Committee for Quality Assurance. The State of Health Care Quality 2008. Washington DC: National Committee for Quality Assurance; 2008. Available from: http://www.ncqa.org/portals/0/ newsroom/sohc/SOHC_08.pdf. Accessed May 17, 2012.

48. Enright P, Quanjer P. Spirometry for COPD is both underutilized and overutilized. Chest. 2007;132(2):368-370.

49. Nelson SB, Thomashaw BM, Enright P, Mannino DM, Nemenoff EK. A tiered economical approach for COPD case-finding in the general population [abstract]. Am J Respir Crit Care Med. 2010;181:A5949.

50. Arne M, Lisspers K, Ställberg B, et al. How often is diagnosis of COPD confirmed with spirometry? Respir Med. 2010;104(4):550-556.

51. Brand PL, Rijcken B, Schouten JP, Koëter GH, Weiss ST, Postma DS Perception of airway obstruction in a random population sample. Relationship to airway hyperresponsiveness in the absence of respiratory symptoms. Am Rev Respir Dis. 1992;146(2):396-401.

52. Kendrick AH, Higgs CM, Whitfield MJ, Laszlo G. Accuracy of perception of severity of asthma: patients treated in general practice. BMJ. 1993;307(6901):422-424.

53. Rennard S, Decramer M, Calverley PM, et al. Impact of COPD in North America and Europe in 2000: subjects' perspective of Confronting COPD International Survey. Eur Respir J. 2002;20(4):799-805.
International Journal of COPD

\section{Publish your work in this journal}

The International Journal of COPD is an international, peer-reviewed journal of therapeutics and pharmacology focusing on concise rapid reporting of clinical studies and reviews in COPD. Special focus is given to the pathophysiological processes underlying the disease, intervention programs, patient focused education, and self management protocols.

\section{Dovepress}

This journal is indexed on PubMed Central, MedLine and CAS. The manuscript management system is completely online and includes a very quick and fair peer-review system, which is all easy to use. Visit http://www.dovepress.com/testimonials.php to read real quotes from published authors. 